\title{
Transient Rotamerism and Photoisomerization Dynamics of trans- and cis-Naphthylstilbene
}

M. T. Quick, ${ }^{1}$ M. Quick, ${ }^{* 2}$ I. N. Ioffe, ${ }^{* 3}$ C. Richter, ${ }^{2}$ R. Mahrwald, ${ }^{2}$ S. I. Druzhinin,,${ }^{4}$ and S. A. Kovalenko ${ }^{2}$

${ }^{1}$ Department for Optics and atomic Physics, Technische Universität, Berlin, Germany

${ }^{2}$ Department of Chemistry, Humboldt-Universität zu Berlin, Germany

${ }^{3}$ Department of Chemistry, Lomonosov Moscow State University, Moscow, Russia

${ }^{4}$ University of Siegen, Department of Chemistry \& Biology, Siegen, Germany

\section{Supporting Information}

Figure S1: $\quad$ Photoisomerization dynamics of $\mathbf{t} \mathrm{N}$ and $\mathbf{c N}$ upon $345 \mathrm{~nm}$ light in $\mathrm{n}$-hexane.

Figure S2: $\quad$ Photoisomerization dynamics of $\mathbf{t N}$ and $\mathbf{c N}$ upon $345 \mathrm{~nm}$ light in $\mathrm{n}$-decane.

Figure S3: $\quad$ Photoisomerization dynamics of $\mathbf{t} \mathrm{N}$ and $\mathbf{c N}$ upon $345 \mathrm{~nm}$ light in $\mathrm{n}$-hexadecane.

Figure S4: $\quad$ Photoisomerization dynamics of $\mathbf{t N}$ and $\mathbf{c N}$ upon $345 \mathrm{~nm}$ light in acetonitrile.

Figure S5: Determination of the Rotamer fluorescence spectra of $\mathbf{t}-S,-A$ and $-S$.

Figure S6: $\quad$ TA spectra of $\mathbf{t} \mathrm{N}$ and $\mathbf{c} \mathrm{N}$ in n-decane upon $345 \mathrm{~nm}$ excitation.

Figure S7: $\quad$ Molecular Structures of $\mathrm{cN}-S$ in the excited $S_{1}$ state. 


\section{Photoisomerization of trans- and cis-naphthylstilbene ( $\mathrm{tN}$ and $\mathrm{cN})$}

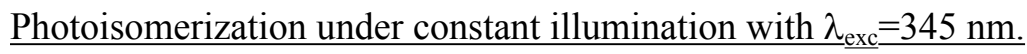

tN is not fully solvable and therefore its extinction is not precisely determinable by conventional methods. Instead, a known mass of $\mathbf{c N}$ is illuminated until photo stationarity with $\mathbf{t} \mathrm{N}$ is established, from where the trans extinction $\varepsilon_{\mathrm{t}}(\lambda)$ is deduced. The extinction spectrum $\varepsilon_{\mathrm{DHP}}(\lambda)$ of the DHP-like photoproduct can be obtained similarly.

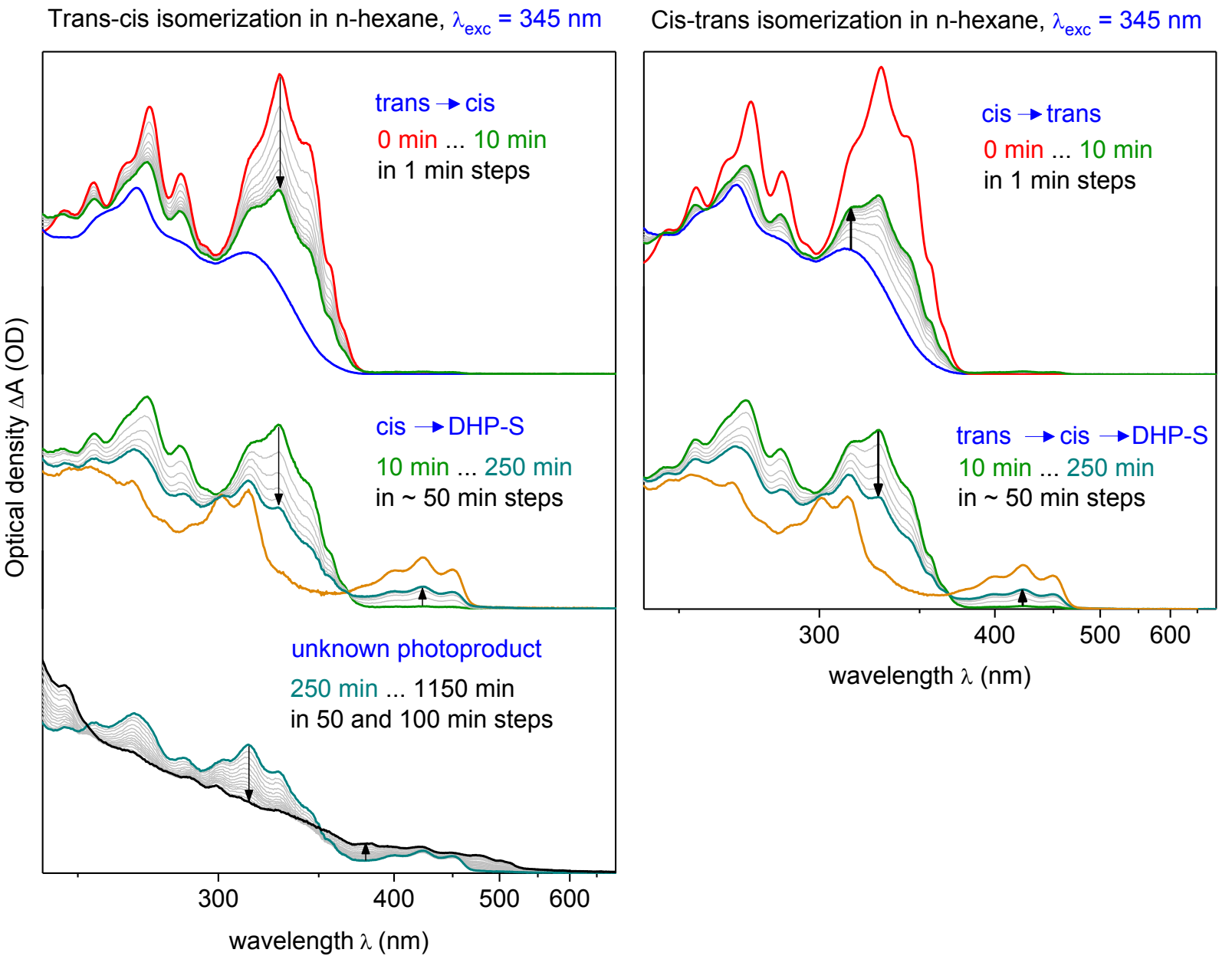

Figure S1. Photoisomerization of $\mathbf{t N}$ and $\mathbf{c N}$ in $\mathrm{n}$-hexane upon $\lambda_{\mathrm{exc}}=345 \mathrm{~nm}$. top, left: starting from a filtered solution of $\mathbf{t N}, \mathbf{c N}$ is produced first within $10 \mathrm{~min}$; middle: at longer illumination up to $250 \mathrm{~min}$, the dihydrophenanthren-like product DHP- $S$ ' is produced. Bottom: further illumination up to $1150 \mathrm{mn}$ decomposes DHP- $S^{\prime}$ resulting in an unknown product. top, right: illumination of $\mathbf{c N}$ firstly produces $\mathbf{t} \mathrm{N}$, and then DHP- $S^{\prime}$ (bottom) indicating a small yield for the ring closure. 

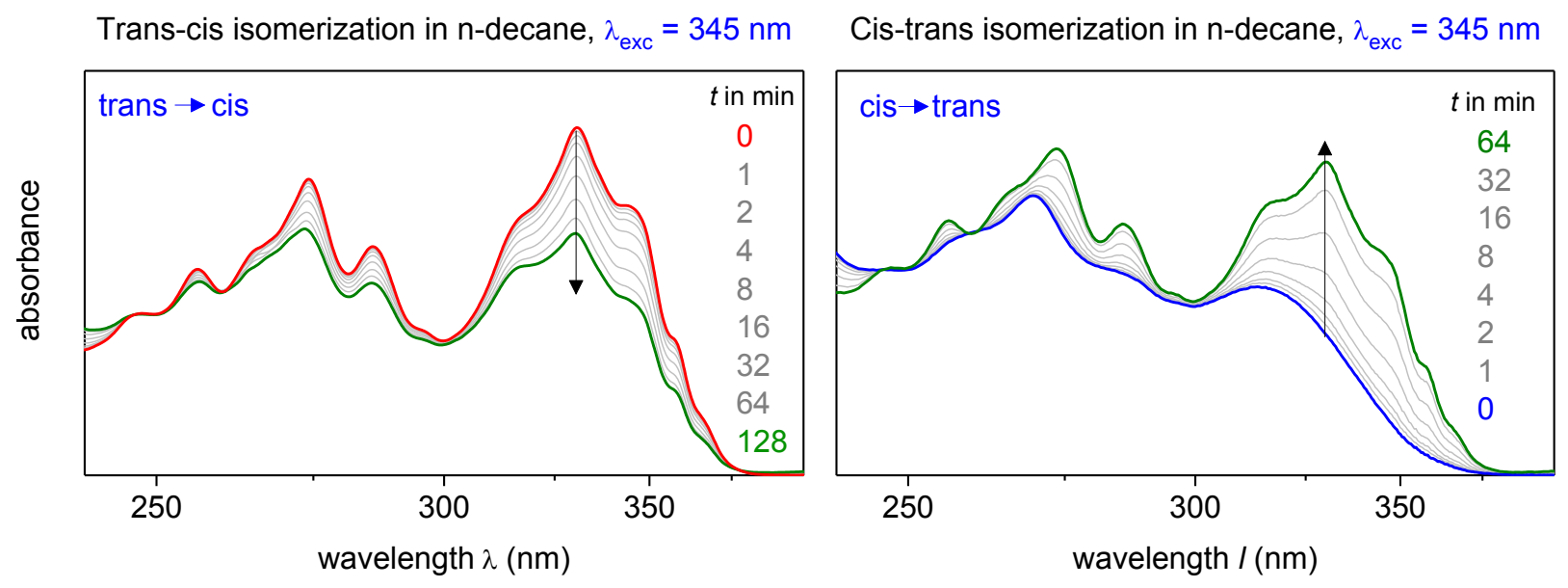

Figure S2. Photosomerization of $\mathbf{t N}$ and $\mathbf{c N}$ in n-decane upon $\lambda_{\mathrm{exc}}=345 \mathrm{~nm}$.

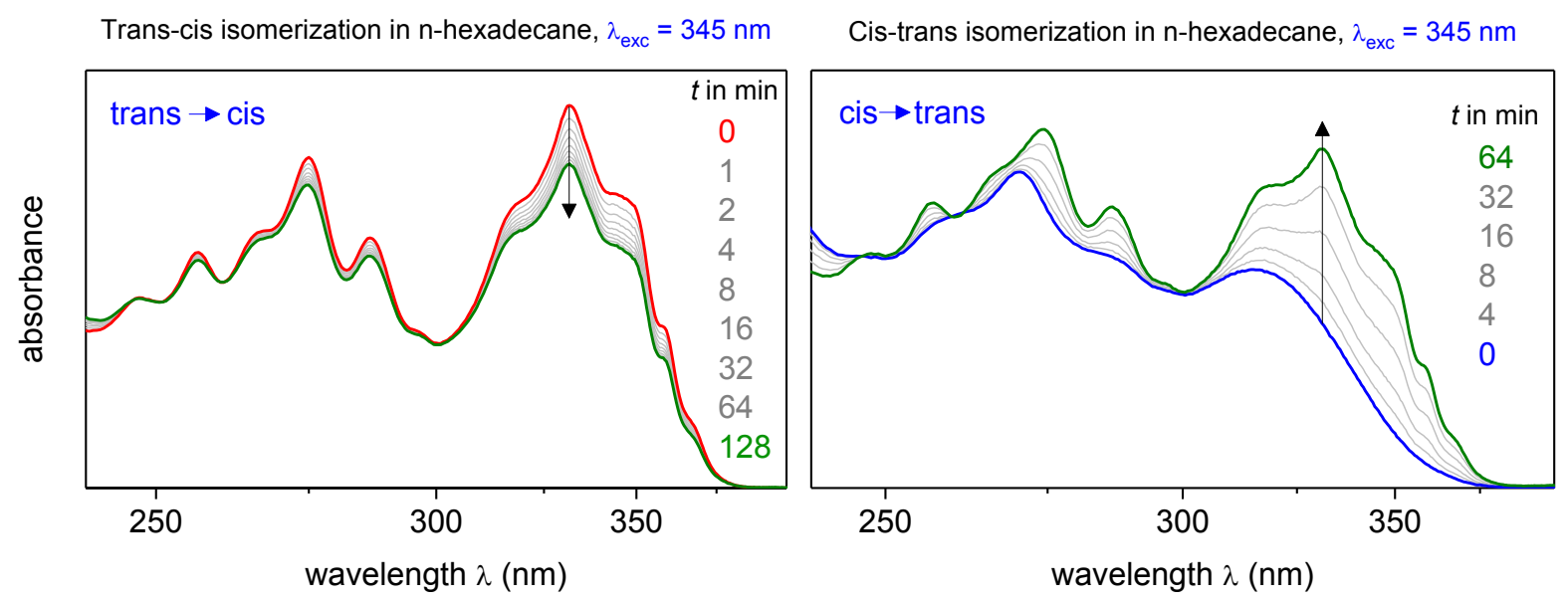

Figure S3. Photosomerization of $\mathbf{t N}$ and $\mathbf{c N}$ in n-hexadecane upon $\lambda_{\mathrm{exc}}=345 \mathrm{~nm}$. 
Trans-cis isomerization in acetonitrile, $\lambda_{\mathrm{exc}}=345 \mathrm{~nm}$

Cis-trans isomerization in acetonitrile, $\lambda_{\text {exc }}=345 \mathrm{~nm}$

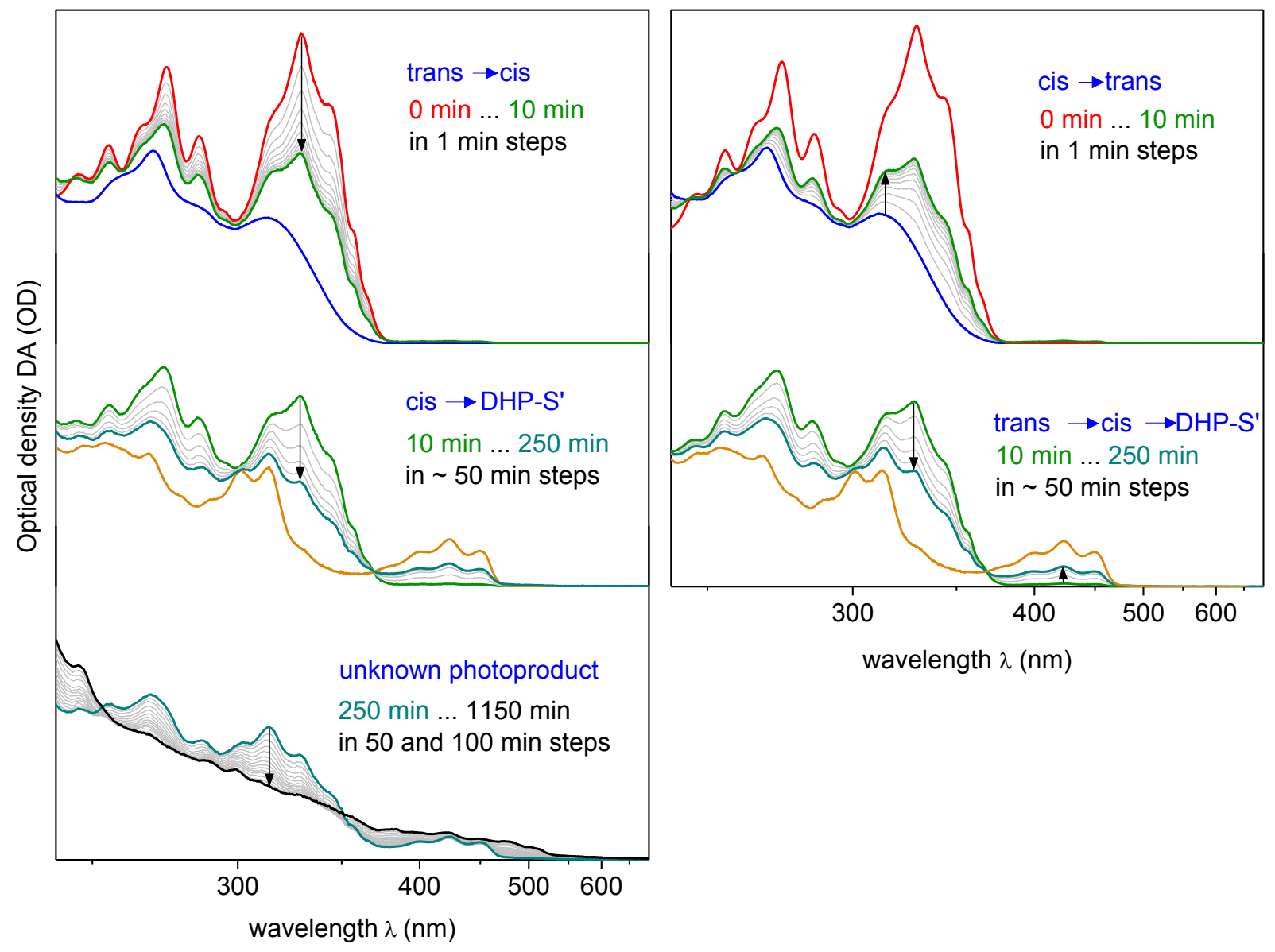

Figure S4. Photosomerization of $\mathbf{t N}$ and $\mathbf{c N}$ in acetonitrile upon $\lambda_{\mathrm{exc}}=345 \mathrm{~nm}$. 


\section{Determination of isomerization quantum yields $\mathbf{s}^{1}$}

\subsection{The kinetic scheme}

The kinetic scheme is expressed as a coupled differential equation that considers a forth- and back reaction between $\mathbf{t N}$ and $\mathbf{c N}$ but also includes reversibility between $\mathbf{c N}$ and DHP (see Scheme S1).

\begin{tabular}{|c|c|c|c|c|c|c|c|c|}
\hline$\dot{C}_{t}$ & $=$ & $-\varphi_{t c}$ & $I_{t}$ & + & $\varphi_{c t}$ & $I_{c}$ & & \\
\hline & $=$ & $\varphi_{t c}$ & $I_{t}$ & - & $\left(\varphi_{c t}+\varphi_{c d}\right)$ & $I_{c}$ & + & $\varphi_{d c}$ \\
\hline & $=$ & & & & $\varphi_{c d}$ & $I_{c}$ & - & $\varphi_{d c}$ \\
\hline
\end{tabular}

Scheme S1: Coupled differential equation that describes the reversibility between $\mathbf{t N}$ and $\mathrm{cN}$ and between $\mathbf{c N}$ and DHP.

$\dot{C}_{x}$ describes the time dependent concentration of compound $\mathrm{x}$ in the mixture and $I_{x}$ represents the photon density per second and volume. $\varphi_{t c}$ and $\varphi_{c t}$ contain the dimensions-less quantum yields for the photo isomerization. The $I_{x}$ that is absorbed by the sample can be expressed as follows:

$I_{x}=\left(I_{0}-I_{0} \cdot 10^{-A(t)}\right) \frac{A_{x}(t) 1}{A(t) V}$

$I_{0}$ is the photon density per second at the initial time, $I_{0} \cdot 10^{-A(t)}$ represents the photon density per second that leaves the sample (Lambert-Beer). Therefore the difference represents the amount that is absorbed by the entire sample. Of these, compound $\mathrm{x}$ absorbs the corresponding amount that can be found by scaling with $\frac{A_{x}(t)}{A(t)}$. The volume $\mathrm{V}$ covers the entire solution inside of the cuvette. This is valid because all molecules enter the irradiated area by convection. Replacing $A_{x}(t)$ by Lambert-Beer's Law and summarizing a few terms to $\mathrm{k}(\mathrm{t})$ gives :

$I_{t, c, d}=\varepsilon_{t, c, d} \cdot C_{t, c, d}(t) \cdot k(t)$

$k(t)=\frac{I_{0} \cdot d}{V} \cdot \frac{\left(1-10^{-A(t)}\right)}{A(t)}$

\subsection{Reduction to equilibrium between cis and trans}

To simplify the coupled equation in Scheme S1 the formation of DHP is neglected. That is almost the case for the measurements performed in n-decane and n-hexadecane, where DHP has not shown up even after two hours of illumination. The photo-stationary state is therefore established only between $\mathbf{t} \mathrm{N}$ and cN (see Scheme S2). Several substitutions on [2] were performed: the $I_{x}$ are replaced by equation [1], terms regarding concentrations are translated to mole-fraction by a division through initial concentrations and each line is expressed by either $X_{t}$ or $X_{c}$. 


$$
\begin{aligned}
& \dot{X}_{t}=-k(t)\left(\varepsilon_{t} \cdot \varphi_{t c}+\varepsilon_{c} \cdot \varphi_{c t}\right) \quad X_{t}+\quad k(t) \cdot \varepsilon_{c} \cdot \varphi_{c t} \\
& \dot{X}_{c}=-k(t)\left(\varepsilon_{t} \cdot \varphi_{t c}+\varepsilon_{c} \cdot \varphi_{c t}\right) \quad X_{c}+k_{t c}
\end{aligned}
$$

Scheme S2: The coupled differential equation now neglects the formation of DHP.

These equations can be solved independently including the following side conditions, exemplarily shown for $X_{c}$ but equivalent for $X_{t}$ :

$\dot{X}_{c, P S S}=0 \rightarrow \frac{\varphi_{t c}}{\varphi_{c t}}=\frac{X_{c, P S S} \cdot \varepsilon_{c}}{\left(1-X_{c, P S S}\right) \cdot \varepsilon_{t}}$

$X_{c, 0}=1$

Equation [4] is useful to express $\varphi_{t c}$ by $\varphi_{c t}$ so that the quantum yield for the cis-trans isomerization can be extracted from the experimental data starting from pure $\mathbf{c N}$. Solving both equations of scheme 2 yields:

$X_{c}(t)=\left(1-X_{c, P S S}\right) \exp \left[-K(t) \frac{\varphi_{c t} \varepsilon_{c}}{1-X_{c, P S S}}\right]+X_{c, P S S}$

$X_{t}(t)=\left(X_{t, 0}-X_{t, P S S}\right) \exp \left[-K(t) \frac{\varphi_{t c} \varepsilon_{t}}{1-X_{t, P S S}}\right]+X_{t, P S S}$

$K(t)=\int_{0}^{t} k\left(t^{\prime}\right) d t^{\prime}$

Equations [6] and [7] are solutions that are obtained independently but they still hold the relation:

$X_{c}(t)+X_{t}(t)=1$

Still, small amounts of DHP are formed eventually even though they are considerably small. They have to be subtracted from the data set and the resulting spectra are rescaled so that equation [9] holds for all times. Equations [6] and [7] need to fit to the experimental by the optimization of $\varphi_{t c}$ and $\varphi_{c t}$ (results in Table 1 of the main text).

\subsection{SVD and kinetic modelling}

In the following section a complete procedure for the simultaneous collection of species associated spectra (SAS) and quantum yields is presented. This time, the formation of DHP is considered here which is already outlined in Scheme S1. That is the case for measurements done in n-hexane and acetonitrile. Little simplifications that facilitate further evaluations are performed and shown in Scheme S3.

$$
\dot{C}_{t}=-\tilde{k} \cdot \varepsilon_{t} \cdot \varphi_{t c} \quad C_{t}+\quad \tilde{k} \cdot \varepsilon_{c} \cdot \varphi_{c t} \quad C_{c}
$$




$$
\begin{aligned}
& \dot{C}_{c}=\tilde{k} \cdot \varepsilon_{t} \cdot \varphi_{t c} \quad C_{t}-\tilde{k} \cdot \varepsilon_{c} \cdot\left(\varphi_{c t}+\varphi_{c d}\right) \quad C_{c}+\tilde{k} \cdot \varepsilon_{d} \cdot \varphi_{d c} \quad C_{d} \\
& \dot{C}_{d}=\quad \begin{array}{lllll}
\tilde{k} \cdot \varepsilon_{c} \cdot \varphi_{c d} & C_{c} & - & \tilde{k} \cdot \varepsilon_{d} \cdot \varphi_{d c} & C_{d}
\end{array}
\end{aligned}
$$

Scheme S3: Similar to Scheme S1 but with time-averaged $k(t)$ to simplify the evaluation.

The time-dependence of $\mathrm{k}(\mathrm{t})$ is rather small and $\tilde{k}$ expresses this constancy. In equation [13] the time weighted integral is calculated.

$\tilde{k}=\frac{I_{0} l}{V t_{n}-t_{1}} \int_{t_{1}}^{t_{n}} \frac{\left(1-10^{-A(t)}\right)}{A(t)} d t$

\subsection{Species associated spectra}

The time-dependent absorption-data is expressed by a matrix $A_{\lambda t}$ where spectral and temporal information is stored in the rows and columns. In principle, each spectrum at any time is a linear combination of the extinction spectra of all participating species, weighted by their actual concentration (see eq. 14).

$A_{\lambda t}=\varepsilon_{\lambda k} C_{k t}=U_{\lambda s} \Sigma_{s s} V_{s t}^{T}$

A singular value decomposition (SVD) is performed which yields three matrices: $U_{\lambda s}, \Sigma_{s s}$ and $V_{s t}^{T}$. For now, $U_{\lambda s}$ and $V_{s t}^{T}$ are matrices that contain spectral and temporal information, respectively, in a rather abstract way. The diagonal matrix $\Sigma_{S S}$ contains the singular values that provide information about the number of participating species. In our case three species contribute to the spectra and the matrices have to be reduced down to this number, symbolically shown in equation [15].

$A_{\lambda t} \approx U_{\lambda k} \Sigma_{k k} V_{k t}^{T}=\left(U_{\lambda k} \sqrt{\Sigma_{k k}}\right)\left(\sqrt{\Sigma_{k k}} V_{k t}^{T}\right)=B_{\lambda k} P_{k t}$

The index $\mathrm{k}$ equals three (not to confuse with the $\mathrm{k}$ from equation 3 ). The matrix $\Sigma_{s s}$ is split up into two matrices and equally distributed to the left and right. Going from abstraction to physical relevance the matrix $P_{k t}$ can be expressed by the product in equation [16]

$P_{k t}=F_{k k} T_{k t}$

$T_{k t}=\left(\begin{array}{ccc}1 & \cdots & 1 \\ e^{-\gamma_{1} t_{1}} & \cdots & e^{-\gamma_{1} t_{n}} \\ \vdots & \ddots & \vdots \\ e^{-\gamma_{k} t_{1}} & \cdots & e^{-\gamma_{k} t_{n}}\end{array}\right)$

in which $T_{k t}$ contains the population curves in form of time-functions that express exponential decay (see eq. 18). Optimizing the rate constants $\gamma_{\mathrm{I}}$ so that equation [17] is fulfilled yield matrix $F_{k k}$. The product $B_{\lambda k} F_{k k}$ now contains the decay associated spectra that describe spectral changes proceeding with $\gamma_{\mathrm{I}}$.

To obtain the SAS a model matrix is postulated that satisfies the relation 
In which $C_{k t}$ contains the population of each contributing species. In turn, the inverse matrix has to be multiplied to the product $B_{\lambda k} F_{k k}$ (see eq. 19).

$$
\begin{aligned}
A_{\lambda t} & =\underbrace{B_{\lambda k} F_{k k} M_{k k}^{-1}}_{\lambda k} \underbrace{M_{k k} T_{k t}} \\
& =\varepsilon_{\lambda k} \quad C_{k t}
\end{aligned}
$$

Reconsidering scheme 3 the matrix representation is depicted in equation [20]

$$
\left(\begin{array}{l}
\dot{C}_{t} \\
\dot{C}_{c} \\
\dot{C}_{d}
\end{array}\right)=\left(\begin{array}{lll}
\frac{\partial \dot{C}_{t}}{\partial C_{t}} & \frac{\partial \dot{C}_{t}}{\partial C_{c}} & \frac{\partial \dot{C}_{t}}{\partial C_{d}} \\
\frac{\partial \dot{C}_{c}}{\partial C_{t}} & \frac{\partial \dot{C}_{c}}{\partial C_{c}} & \frac{\partial \dot{C}_{c}}{\partial C_{d}} \\
\frac{\partial \dot{C}_{d}}{\partial C_{t}} & \frac{\partial \dot{C}_{d}}{\partial C_{c}} & \frac{\partial \dot{C}_{d}}{\partial C_{d}}
\end{array}\right)\left(\begin{array}{l}
C_{t} \\
C_{c} \\
C_{d}
\end{array}\right)=J_{k k}\left(\begin{array}{l}
C_{t} \\
C_{c} \\
C_{d}
\end{array}\right)
$$

Where $J_{k k}$ is the Jacobian matrix whose eigenvalues are the rate constants. In the course of solving eq. [20] for the $C_{k}$ one will find that $M_{k k}$ is given by the product

$M_{k k}=W_{k k} \operatorname{diag}\left(W_{k k}^{-1} C_{k 1}\right)$

Here $C_{k 1}$ is a column vector containing the initial concentrations of each component. The symmetric matrix $W_{k k}$ is holding the Eigenvectors of $J_{k k}$ column-wise. Since this system is under determined (i.e.

there are more $\tilde{k} \varepsilon_{i} \varphi_{i j}$ than $\gamma_{k}$ ), it is necessary to define ratios $\frac{\varphi_{t c}}{\varphi_{c t}}$ and $\frac{\varphi_{d c}}{\varphi_{c d}}$ as additional optimization parameters. The ratios were adjusted to yield the best structural agreement between the measured $\varepsilon(\lambda)$ and the SAS of $\mathbf{t} \mathrm{N}$ and $\mathbf{c N}$. One criteria for the SAS of DHP was that almost no structure is located between 320 - $370 \mathrm{~nm}$. Quantum yields of photoreactions are collected in Table 6 in the main text.

\section{Rotamer Fluorescence Spectra}

In order to obtain the rotamer fluorescence spectra, fluorescence spectra measured over different excitation wavelengths are averaged and offset against each other. Specifically, first, average spectra are formed over the excitation regions $370-378 \mathrm{~nm}$ and $364-370 \mathrm{~nm}$. In the former case, the rotamers of symmetry $A$ and $S$ are in the same ratio. From this, the second average spectrum is subtracted (after scaling with a factor), which mainly contains contributions from $\mathbf{t} \mathrm{N}-A$. The result is the pure fluorescence spectrum of the rotamer $\mathbf{t} \mathrm{N}-S^{\prime}$. Symmetry $A$, on the other hand, is obtained when the contribution of the symmetry $S^{\prime}$ is subtracted completely from the second average spectrum (364 - $\left.370 \mathrm{~nm}\right)$. For the fluorescence spectrum of the symmetry $S$, a further average spectrum is formed, namely over the range 340-348 nm. This contains $\mathbf{t N}-S$ and $-A$ in equal parts. The newly obtained fluorescence spectrum of symmetry $A$ is subtracted from this with a scaling factor until the fluorescence spectrum of $\mathbf{t N}-S$ remains (see Fig. S5). 

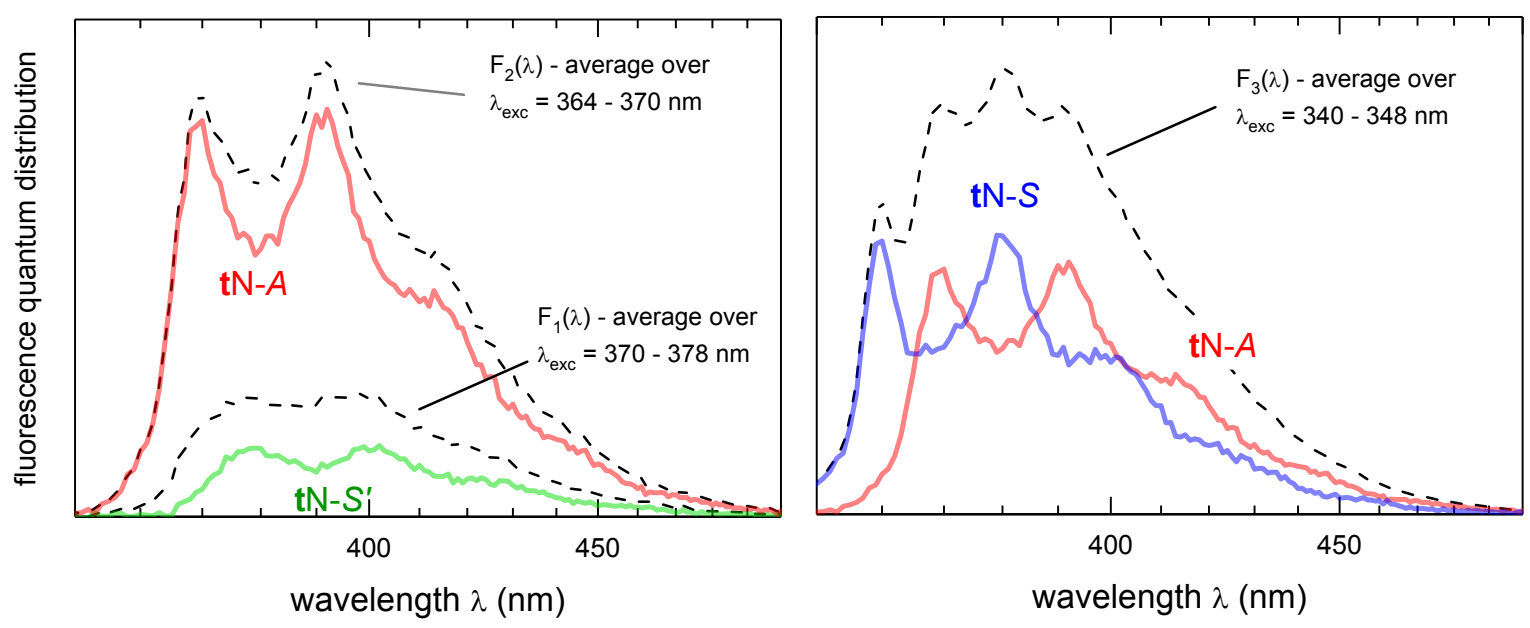

Figure S5. Procedure to obtain the rotamer fluorescence spectra from the measured spectra. The average spectra $F_{1}(\lambda)$ and $F_{2}(\lambda)$ obtained over the excitation wavelength ranges $370-378 \mathrm{~nm}$ and 364 $370 \mathrm{~nm}$, respectively, contain the rotamer fluorescence spectra of $\mathbf{t} \mathrm{N}-A$ and $-S$ ' in different weights. Careful subtraction of $\mathrm{F}_{2}(\lambda)$ from $\mathrm{F}_{1}(\lambda)$ eliminates all contributions from $\mathbf{t} \mathrm{N}-A$, but leaves emission from pure $\mathrm{tN}^{-} S^{\prime}$ (green). The main criterion for subtraction is that the high frequency vibrational band of the $\mathbf{t} \mathrm{N}-A$ emission vanishes completely. The pure emission from $\mathbf{t} \mathrm{N}-S^{\prime}$ is now subtracted accordingly from $\mathrm{F}_{2}(\lambda)$ until the pure emission from $\mathbf{t} \mathrm{N}-A$ (red) remains. Similarly, an average fluorescence spectrum $\mathrm{F}_{3}(\lambda)$ is calculated from the excitation wavelength range $340-348 \mathrm{~nm}$ which contains only contribution of $\mathbf{t} \mathrm{N}$ $S$ and $-A$. Subtraction of the $\mathbf{t} \mathrm{N}-A$ fraction leaves the pure emission spectrum from $\mathbf{t} \mathrm{N}-S$ (blue). 


\section{TA spectra of $\mathrm{tN}$ and $\mathrm{cN}$ in n-decane}
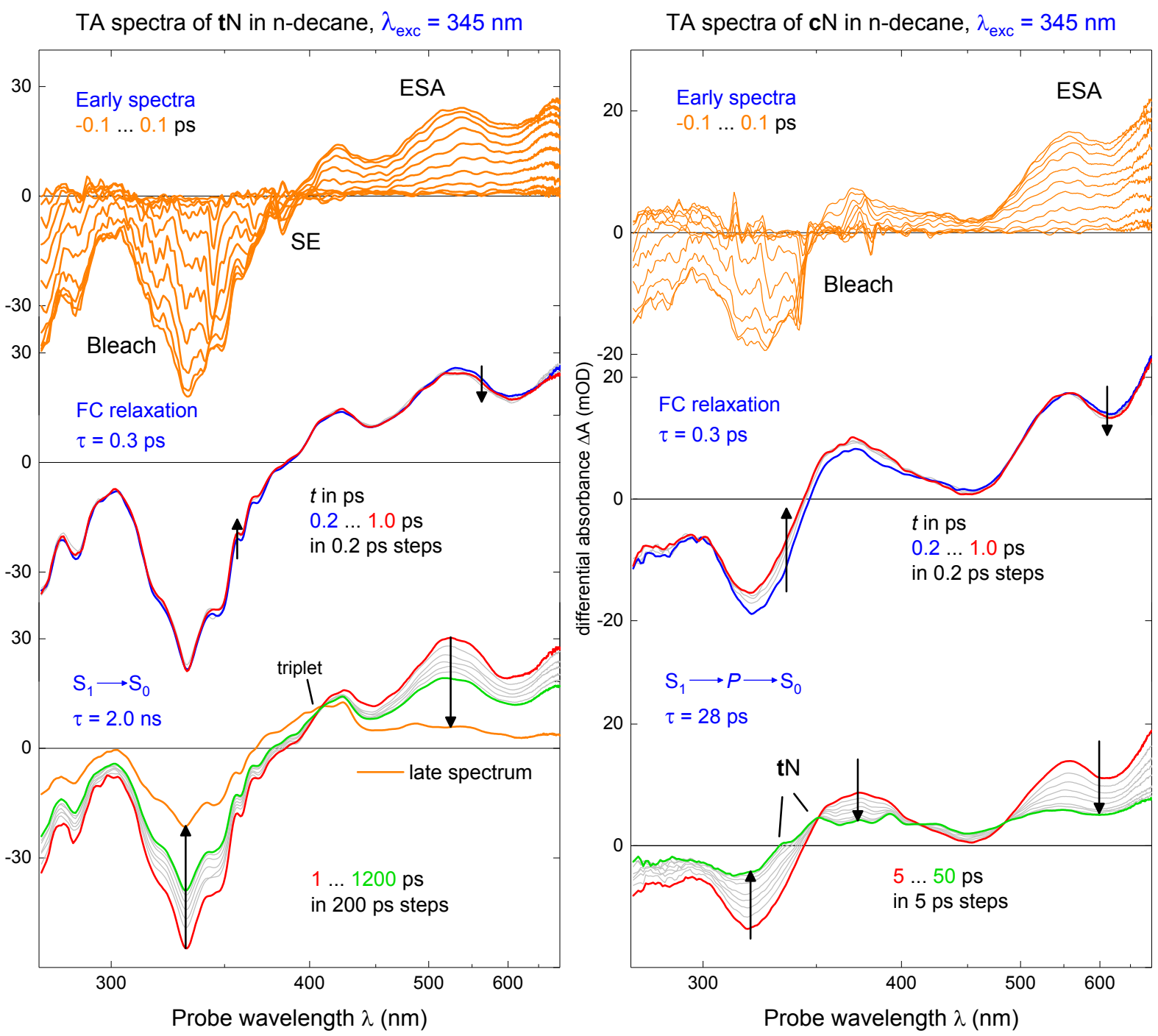

Figure S6. left - TA spectra of $\mathbf{t N}$ are measured in n-decane after excitation at $345 \mathrm{~nm}$. At this wavelength, the rotamer ratio is not altered in the ground state, which means that the process of rotamerism is not visible in the TA spectrum. The excited state lifetime does not depend on the solvent viscosity, suggesting the low importance of trans-cis isomerization compared to radiative deactivation. The fluorescence lifetimes have been determined $2.1 \mathrm{~ns}$. Triplet ESA can be seen at $420 \mathrm{~nm}$ after the $\mathrm{S}_{1}$ state has decayed. right - TA spectra of $\mathbf{c N}$ are measured in n-decane upon $345 \mathrm{~nm}$ excitation. After FC relaxation, ESA decays with a 28 ps time constant as the cis-site of $\mathrm{S}_{1}$ is depopulated via isomerization to $P$. At the same time, bleach vanishes, which denotes that the $P$-state lives too short to be resolved and $\mathrm{S}_{0}$ recovers instantly. 


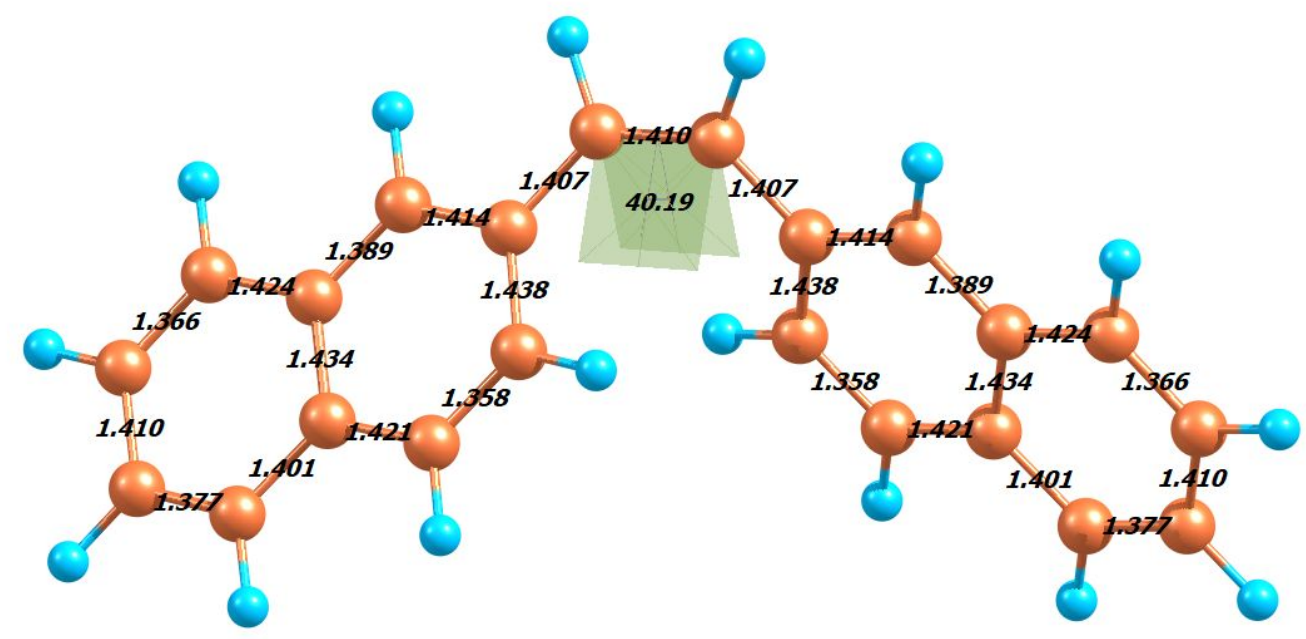

cN-S: stationary point in the $S_{1}$ state

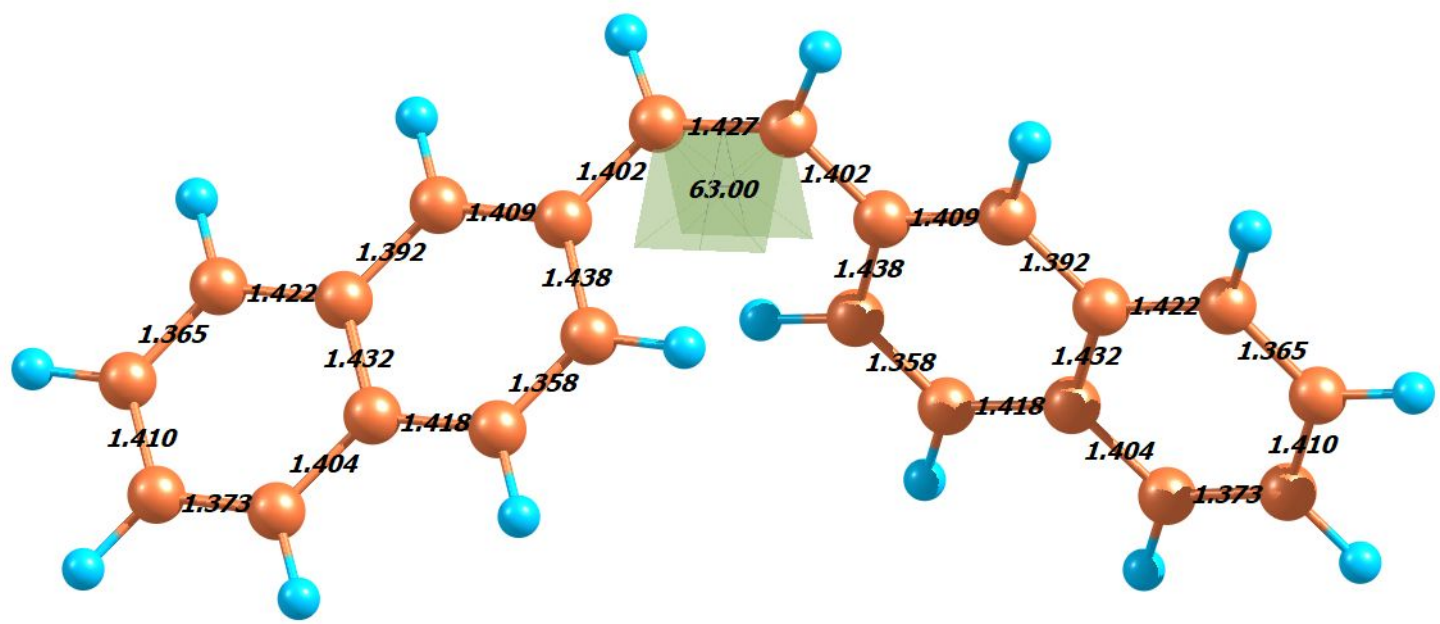

cN-S: transition state for the $S_{l} \rightarrow P$ twisting

Figure S7. Molecular Structures of $\mathrm{cN}-S$ in the excited $S_{1}$ state. 


\section{References}

(1) Mauser, H.; Gauglitz, H. Theoretical Fundamentals and Applications. Photokinetics 1998, Vol. 36 (1 $1^{\text {st }}$ Edition). 